\title{
Expression Profile of Signal Transduction Components in a Sugarcane Population Segregating for Sugar Content
}

\author{
Juliana de Maria Felix • Flávia Stal Papini-Terzi • Flávia Riso Rocha • \\ Ricardo Zorzetto Nicoliello Vêncio • Renato Vicentini • Milton Yutaka Nishiyama Jr • \\ Eugênio César Ulian • Gláucia Mendes Souza • Marcelo Menossi
}

Received: 14 May 2009 /Accepted: 22 June 2009 / Published online: 15 July 2009

(C) Springer Science + Business Media, LLC 2009

\begin{abstract}
Sucrose is the major product of photosynthesis in many higher plants. It is transported from the source tissue through the phloem to various sink tissues to support plant growth, development and reproduction. Knowledge on the
\end{abstract}

Communicated by: Ray Ming

Juliana de Maria Felix and Flávia Stal Papini-Terzi contributed equally to this work.

Electronic supplementary material The online version of this article (doi:10.1007/s12042-009-9031-8) contains supplementary material, which is available to authorized users.

R. Vicentini

Centro de Biologia Molecular e Engenharia Genética,

Universidade Estadual de Campinas,

Caixa Postal 6010,

Campinas, SP 13083-875, Brazil

J. de Maria Felix $\cdot$ R. Vicentini $\cdot$ M. Menossi $(\bowtie)$

Instituto de Biologia, Departamento de Genética,

Evolução e Bioagentes, Universidade Estadual de Campinas,

Caixa Postal 6109,

Campinas, SP 1303-862, Brazil

e-mail:menossi@unicamp.br

F. S. Papini-Terzi $\cdot$ F. R. Rocha $\cdot$ R. Z. N. Vêncio

M. Y. Nishiyama Jr • G. M. Souza

Instituto de Química, Departamento de Bioquímica,

Universidade de São Paulo,

Av. Prof. Lineu Prestes, 748,

São Paulo, SP 05508-900, Brazil

E. César Ulian

Centro de Tecnologia Canavieira-CTC,

Caixa Postal 162,

Piracicaba, SP 13400-970, Brazil

Present Address:

J. de Maria Felix

Centro de Tecnologia Canavieira, CTC,

Piracicaba, SP 13400-970, Brazil signal transduction pathways involved in sucrose synthesis in mature leaves is limited. Using a microarray approach, we analyzed the expression profiles of 1920 sugarcane genes encoding signal transduction elements, transcription factors and stress-related proteins. We used individuals from a population segregating for sugar content and gene expression profiles were obtained from seven individuals with highest and seven with lowest sugar content. Surprisingly, from the 24 differentially expressed genes, 19 were more expressed in plants containing low-sugar content. Three of these genes encoded 14-3-3 like proteins, which have been found to reduce sucrose phosphate synthase (SPS) activity. Another encoded an SNF1-related protein similar to a protein kinase that phosphorylates SPS in vitro making it a target for the interaction with 14-3-3 proteins. The up-regulation of eight stress related genes in the lower sugar content plants supports a view that sugar levels modulate a complex signal transduction network that seems to involve responses that are related to stress. Evidence that hormone signaling is related to the sucrose content was also found. These data reinforced the usefulness of genomic approaches to uncover how sucrose metabolism can be regulated in sugarcane.

Keywords cDNA microarray - Gene expression .

Signal transduction $\cdot$ Sugarcane $\cdot$ Sucrose synthesis

$\begin{array}{ll}\text { Abbreviations } & \\ \text { EST } & \text { Expressed Sequence Tag } \\ \text { HPLC } & \text { High Performance Liquid Chromatography } \\ \text { HPAEC- } & \text { High Performance Anion Exchange } \\ \text { PAD } & \text { Chromatography } \\ \text { SAS } & \text { Sugarcane Assembled Sequence }\end{array}$




\section{Introduction}

Plants synthesize carbohydrates in leaves by photosynthetically fixing atmospheric $\mathrm{CO}_{2}$. In $\mathrm{C}_{4}$ plants, like sugarcane, maize and sorghum, the $\mathrm{CO}_{2}$ fixation occurs in two photosynthetic cell types: mesophyll cells and bundle sheath cells. Mesophyll cells carry out the initial steps of $\mathrm{CO}_{2}$ fixation via the enzyme phosphenolpyruvate (PEP) carboxylase to produce the four-carbon organic acid oxaloacetate. In the bundle sheath cells, the $\mathrm{C}_{4}$ acid is decarboxylated and Rubisco refixes the resulting $\mathrm{CO}_{2}$ in the photosynthetic carbon reduction (PCR) cycle (reviewed by Lunn and Furbank [25]).

Sucrose is the major form in which carbohydrate is translocated from leaves to the rest of the plant, to supply carbon and energy for growth and the accumulation of storage reserves. After synthesized, it can be either stored temporarily in the vacuole or transported over long distance in solution in the phloem sap. Photosynthetically tissues, like mature leaves, are net exporters of sugars and are known as 'carbon sources' or source tissues. Heterotrophic cells in roots, reproductive structures, storage and developing organs rely on a supply of sugars for their nutrition; these are known as 'carbon sinks' or sink tissues (net importers). Sucrose itself is the major storage reserve in some plants, for example in sugarcane (Saccharum spp.) stems, sugarbeet (Beta vulgaris) roots and the fruits of many species.

There is a growing interest in the tropical crop sugarcane because ethanol and biomass are important renewable biofuel sources. Moreover it is of great economic interest, contributing to about two thirds of the world's raw sugar production [41]. Due to its unique capacity of storing sucrose in the stems, sugarcane is an interesting model for studies on sugar synthesis, transport and accumulation [29]. Sucrose metabolism components and regulators are likely to be key players in determining sugarcane sucrose yield $[25,31]$.

In addition to being an important carbon reserve in different organs, such as stems, tubers and fruits, sucrose also helps to protect plants from environmental stresses as, for example, cold and drought [46]. The accumulation of sucrose and other low- molecular-mass compounds under stress conditions is often regarded as an adaptive mechanism to maintain cell turgor and to protect the structure and function of proteins and membranes.

Moreover, it has been recognized that sucrose also acts as a signal compound, affecting a variety of physiological processes, such as photosynthesis, source and sink metabolism and defense responses [13, 23, 28, 35, 43, 46]. Metabolism control involves the coordinated regulation of genes and enzymes at the level of transcription, translation, post-translational modification and protein turnover. The carbon metabolite signaling pathways cross-talk with other pathways, including hormonal responses, cell cycle control and nitrogen response systems, amongst others [15]. Whereas the effect of sugars on gene regulation is well established, the nature of the signals and the molecular mechanisms involved in sugar perception and intracellular signal transmission are largely unknown. Therefore, understanding sucrose synthesis in sugarcane at the transcriptional level, and finding the genes coding for proteins associated with sugar accumulation would be of great value for the long-term success of varietal improvement.

Sugarcane is a complex polyploid grass with commercial varieties derived from conventional breeding. Recent yield data indicate that such technology may be reaching its limit with respect to increases in sugar productivity. It would be highly advantageous to have genes associated with desirable traits targeted for directed improvement of the varieties. A useful strategy for target-gene identification has been denominated "genetical genomics". First introduced by Jansen and Nap [21], this method aims to apply large-scale analysis of gene expression to a segregated population. The use of cDNA microarrays to evaluate an $F_{1}$ sugarcane population that segregates for a certain trait may provide more insight into plant signaling and gene function than classical mutagenesis studies [30]. Recently, Casu et al. [7] and Papini-Terzi et al. $[37,38]$ used this strategy to identify genes associated with high sucrose accumulation in sugarcane stem. The genomics approach has been the method of choice in the search for coarse regulatory mechanisms of sugarcane sucrose accumulation and signaling [3-7, 28, 37, 38]. However, most of the studies on the sugarcane transcriptome have focused primarily on the sugarcane stem during vegetative growth, i.e., on internodes actively accumulating sucrose.

In this study we used a bulk segregant analysis as a first step towards a "genetical genomics" approach for the identification of genes whose differential expression levels correlated with high or low sugar contents in a segregating sugarcane population. Microarrays containing 1920 signal transduction-related ESTs as well as transcription factors and stress-related elements were used to measure relative gene expression. A total of 24 SAS (Sugarcane Assembled Sequences) were defined as differentially expressed. These genes also had differential expression along the growing season and in different tissues. The role of these genes in sugar perception and intracellular signal transmission mechanisms in regulating sucrose metabolism and accumulation are discussed

\section{Results}

Sugar Content in a Field-Grown $\mathrm{F}_{1}$ Segregant Progeny

In order to assess differences in gene expression associated with sugar content, individuals from a sugarcane progeny 
contrasting for sucrose content were chosen for the analysis. The plant material used was a field-grown F1 progeny selected from a cross between the sugarcane varieties SP 80-180 and SP 80-4966. The parental are divergent for sucrose content and differ by 3.07 points in their Brix content (data not shown). From a total of 498 individuals, seven plants with the highest (7HS) and seven with the lowest (7LS) sugar contents were picked out. Figure 1 shows the average values and standard deviations for the soluble solids content (Brix) of the most mature internode of these group of plants measured throughout the growing season $(6,7,9,11$ and 13 months after planting). Figure $2 \mathrm{a}$ shows the average values taken from leaves of three individual clones of each segregated plants (HS- high and LS - low sugar content) chosen for xylitol, mannitol, trehalose, arabinose, galactose, glucose, fructose, maltose and sucrose contents, measured by the HPLC and HPAEC-PAD method. Figure $2 b$ shows the measurement of the same sugars for the most mature internode of the same three individuals clones of each segregated plants used for leaves. We can observe that sucrose levels in mature leaves are in agreement with the Brix content and the sucrose levels in mature internode. Differences in gene expression in mature leaves can be associated with the sugar content in these plants.

\section{Differential Gene Expression in Mature Sugarcane Leaves}

Mature leaves from high and low sugar content plants were collected 9 months after planting since at this age the great difference in sugar content was observed between the two

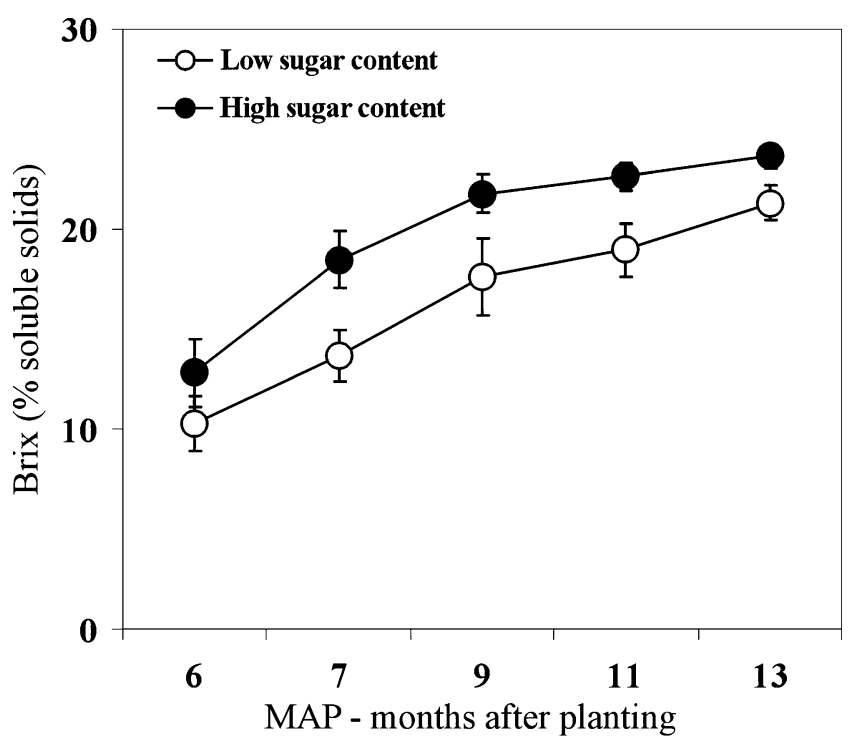

Fig. 1 Sugar content throughout the growing season in the extreme individuals of a sugarcane segregated population. The Brix (soluble solids) values of the most mature internodes of each sugarcane segregant plant were measured throughout the growing season. Average Brix values and standard deviations for the seven individuals with the highest or lowest sugar contents are shown for the times indicated segregant samples (Fig. 1). To evaluate differences in gene expression levels between individuals from these two populations we bulked the leaf tissues from seven plants representing the individuals with the highest sugar content (HS) and lowest sugar content (LS). The RNA from these bulks was further hybridized to cDNA microarrays, in a kind of Bulk Segregant Analysis as a first approach to a genetical genomics analysis.

Twenty-four ESTs were differentially expressed in the two groups. The putative biological functions associated with these ESTs are shown in Table 1. Five transcripts were enriched in the mature leaves of the higher sugar content plants. These encoded an omega-3 fatty acid desaturase (FAD8), two sequences with no hits in the public databases ('no match'), a putative receptor-like serine/threonine kinase (ScBAK1, [54]) and a Myb domain transcription factor LHY/ CCA1. Nineteen transcripts were enriched in the mature leaves of the lower sugar content plants. These encoded three 14-3-3 like proteins, two proteins of the inositol metabolism (O-methyltransferase and 1,4,5-trisphosphate phosphatase), a SNF1-related protein (SnRK1), a putative protein with an unknown function, eight stress-related proteins, two transcription factors, a F-box TIR-1 and one putative protein with no match in the GenBank database. It is interesting to note that these genes encoded cellular components of various functional categories, including signaling (ScBAK1, SnRK, 14-3-3), transcription (tubby, DP transcription factor) and stress responses (drought and cold response, wound induced protein, dehydrin, tonoplast intrinsic protein). This indicates that the modulation of sucrose content relies on several metabolic processes, including the perception of stress signals and the regulation of gene expression.

Gene Expression Validation by RNA-blot and Analysis in Plants Throughout the Growing Season

Three genes with greater expression in the higher sugar content plants (encoding omega-3 fatty acid desaturaseFAD8, no match protein and Myb-repeat transcription factor) and three with increased expression in the lower sugar content plants (encoding SNF1-related protein and dehydrin) were analyzed by RNA-blots. Total RNA from each of three sugarcane individuals was used to provide replication for the gene expression profiles observed in the microarray hybridization. Figure 3 shows that the microarray data was confirmed in all three different sugarcane plants collected 9 months after planting, with only a single exception in the case of the no match protein gene, indicating high consistency between the two data sets.

To identify the gene expression trends throughout the growing season, the mRNA levels for the same six genes were determined in the 7HS and 7LS pools collected 6, 7, 9, 11 and 13 months after planting (Fig. 4). The inset graph 


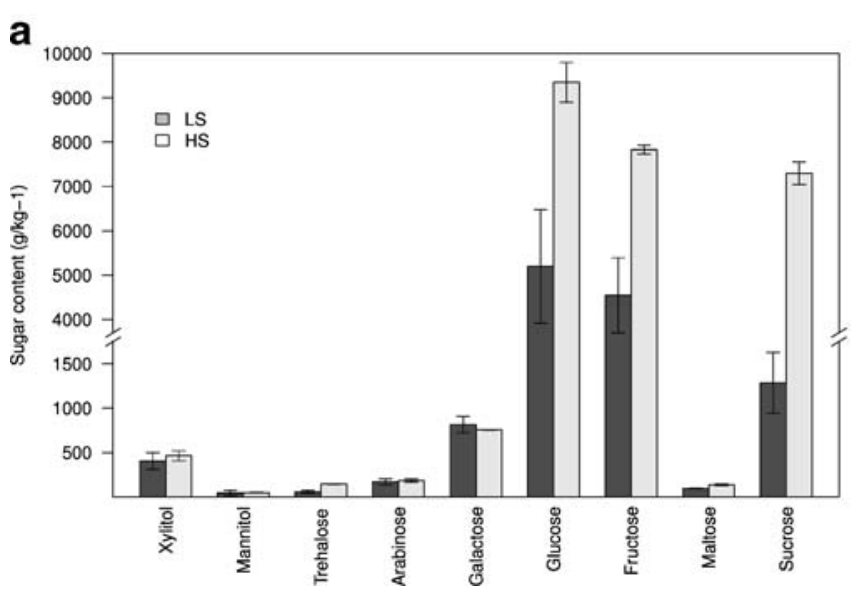

Fig. 2 Distributions of sugars in leaves and internodes of sugarcane individuals by HPLC analysis. Three individual clones of each segregant plants (HS - high and LS - low sugar contents) were chosen to determine the xylitol, mannitol, trehalose, arabinose, galactose, glucose, fructose, maltose and sucrose contents (g kg-1) by HPLC and

represents the expression profile of each gene plotted for each group. The three genes found to be enriched in the higher sugar content plants were consistently differentially expressed throughout the growing season (Fig. $4 \mathrm{a}-\mathrm{c}$ ). The genes with more transcripts in the lower sugar content plants showed a less consistent pattern (Fig. 4d-e). All of them were differentially expressed in the plants at 9 months after planting, confirming the expression observed by microarrays, but only the one encoding dehydrin, a stressrelated protein (Fig. 4 e) had a more consistent pattern throughout the growing season.

Finally, the spatial profile of these ESTs was analyzed, comparing their expression in the source (mature leaf) and sink (immature leaf, immature internode, root, lateral bud and flower) tissues of a commercial sugarcane variety (Fig. 5). The mRNA of FAD8 accumulated at high levels in immature leaves and immature internodes, at a lower level in mature leaves and at very low levels in roots, lateral buds and flowers. Similar patterns were observed for no match protein with preferential expressions in mature leaves and no expression, or a very weak signal, in the other tissues analyzed. The LHY/CAA1 transcript was expressed in all tissues analyzed, but accumulated to high levels in mature and immature leaves, lateral buds and flowers. A similar pattern was observed for SNF-1 related protein and dehydrin, with higher expression levels in immature leaves, immature internodes, lateral buds and flowers.

\section{Discussion}

Gene regulation is based on sensing different signals or stimuli, which are transmitted through a signaling pathway, finally leading to an increase or decrease in transcription. In b

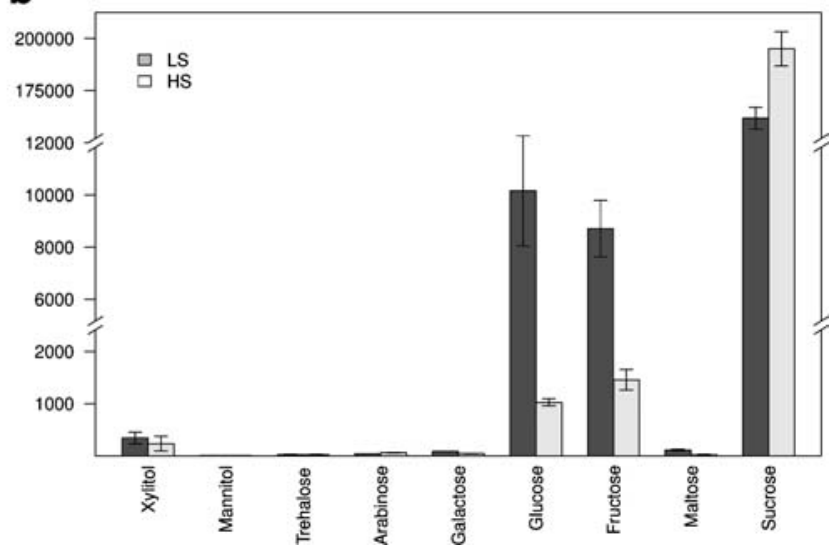

HPAEC-PAD analysis. The average values and standard deviations for the three individuals are shown for the sugars indicated. Distributions of sugars in leaves (a) and the most mature internode (b) of sugarcane individuals by HPLC analysis

sugar signaling, the first step is to sense the nature and level of the specific sugar. While elevated cellular levels of sugar up-regulate genes involved in the synthesis of polysaccharides, storage proteins and pigments, as well as in genes associated with defense responses and respiration, sugar deprivation enhances the expression of genes involved in photosynthesis and resource remobilization, such as the degradation of starch, lipid, and protein $[17,22,56]$. Although the regulatory effect of sugars on photosynthetic activity and plant metabolism has long been recognized, the concept of sugars as central signaling molecules is relatively new (reviewed by Rolland et al. [44]).

Genome-wide expression analysis using cDNA microarray has been applied to the discovery of new insights into the mechanisms by which sugar-response pathways interact with other pathways. Price et al. [42] used this approach to determine the effect of glucose and inorganic nitrogen on gene expression on a global scale in Arabidopsis thaliana. Glucose regulated a broad range of genes, including genes associated with carbohydrate metabolism, signal transduction and metabolite transport. In addition, a large number of stress responsive genes were also induced by glucose, indicating a role for sugar in environmental responses. Similar results were obtained using rice (Oryza sativa) cell cultures, where the transcription rate and mRNA stability were shown to be affected by sugars [17], illustrating a diverse role of sugar in gene regulation. In a microarray study measuring the effects of sucrose and light on 8,000 unique Arabidopsis targets revealed that genes associated with metabolism, protein synthesis/modification and energy were over represented when compared to genes unaffected by the treatments [50]. In a recent study using ATH1 arrays, Osuna et al. [35] identified many genes related to signal transduction like receptor kinases, soluble protein kinases 
Table 1 Sugarcane genes showing differential expression between high and low sugar content populations

\begin{tabular}{|c|c|c|c|c|c|c|}
\hline Category & Acession of $\mathrm{SAS}^{\mathrm{a}}$ & Description of homologue ${ }^{b}$ & Acession of homologue ${ }^{c}$ & E Value $^{\mathrm{d}}$ & $\operatorname{High}^{\mathrm{e}}$ & $\operatorname{High}^{\mathrm{f}}$ \\
\hline \multicolumn{7}{|c|}{ Enriched expression in the high sugar content population } \\
\hline Hormone biosynthesis & CA079174 & Omega-3 fatty acid desaturase-FAD 8 & T01696 & $1 \mathrm{e}-104$ & $1.88 *$ & \\
\hline No matches & CA116458 & No matches & & & $1.89 *$ & \\
\hline No matches & CA275224 & No matches & & & 1.93 & \\
\hline Receptors & CA156919 & RLK undefined with LRR-unclassified & CAB51480 & $1 \mathrm{e}-113$ & 1.64 & \\
\hline Transcription & CA190110 & LHY/CAA1 & XP_480189.1 & $9 \mathrm{e}-69$ & $1.79 *$ & \\
\hline \multicolumn{7}{|c|}{ Enriched expression in the low sugar content population } \\
\hline Adapters & CA146811 & 14-3-3 proteins & AAP48904 & $7 e-140$ & & 1.81 \\
\hline Adapters & CA132593 & 14-3-3 proteins & BAB11739 & $2 \mathrm{e}-80$ & & 2.26 \\
\hline Adapters & CA133114 & 14-3-3 proteins & AAP48904 & $1 \mathrm{e}-117$ & & 1.90 \\
\hline Inositol & CA125200 & Caffeic acid 3-O-methyltransferase & AAQ67347 & 0.0 & & 1.61 \\
\hline Inositol & CA185029 & Inositol-4,5-trisphosphate phosphatase & XP_475767 & $8 \mathrm{e}-62$ & & 3.07 \\
\hline Protein kinases & CA279976 & SNFI-related & CAA73067 & $2 \mathrm{e}-73$ & & $2.28 *$ \\
\hline Putative protein & CA127148 & Putative CGI-94 protein & BAD68235 & $8 \mathrm{e}-96$ & & 1.69 \\
\hline Stress & CA122463 & Dehydrin & AAA33480 & $6 e-48$ & & $1.81 *$ \\
\hline Stress & CA160294 & Low temperature induced (LTI) & AAT37942 & $6 e-24$ & & 2.40 \\
\hline Stress & CA186860 & Low temperature induced (LTI) & AAV88601 & $7 e-18$ & & 2.65 \\
\hline Stress & CA239336 & Reversibly glycosylated polypeptide & XP_479089 & $7 e-18$ & & 2.65 \\
\hline Stress & CA119392 & Tonoplast intrinsic protein & AAC09245 & $6 e-102$ & & 1.90 \\
\hline Stress & CA124270 & Dehydrin & AAB05927 & $7 e-20$ & & 2.57 \\
\hline Stress & CA135201 & Ribonuclease & AAS01727 & $1 \mathrm{e}-106$ & & 2.38 \\
\hline Stress & CA127342 & Wound-induced & CAA42537 & $2 \mathrm{e}-17$ & & 2.03 \\
\hline Transcription & CA110838 & DP transcription factor & AA072671 & $5 e-109$ & & 1.86 \\
\hline Transcription & CA093881 & Tubby-like protein 7 & AAM18187 & $1 \mathrm{e}-71$ & & 1.94 \\
\hline Ubiquitination & CA096709 & F-box containing protein TIR1-like & XP_467902 & $1 \mathrm{e}-173$ & & 1.98 \\
\hline Unknown & CA298983 & Unknown protein & XP_467976 & $9 e-36$ & & 3.62 \\
\hline
\end{tabular}

${ }^{a}$ Acession number of Sugarcane Assembled Sequences; ${ }^{b}$ The description indicates the putative function of the gene products expected from the similarity sequences by searches using the BlastX algorithm [1] and the corresponding SUCAST category; ${ }^{\mathrm{c}}$ The accession number of the homologue in the NCBI public database; ${ }^{\mathrm{d}} \mathrm{E}$ value; ${ }^{\mathrm{e}}{ }_{\mathrm{f}}^{\mathrm{f}}$ Fold increase in expression observed for these ESTs in a high $\left({ }^{\mathrm{e}}\right)$ or low $\left({ }^{\mathrm{f}}\right)$ sugar content plants. Asterisks represent ESTs that were validated by RNA-blots

and phosphatases, MAP kinase pathway components, calcium-binding proteins and G-proteins that presented alterations of their transcript levels in C-starved seedlings, which are reversed by sucrose addition.

Here we report a microarray analysis of 1920 sugarcane genes encoding signal transduction elements, transcription factors and stress-related proteins. The expression profile of these genes in mature leaves of a sugarcane population segregating for sugar content was analyzed and putative targets for molecular-assisted varietal improvement identified. Possible roles of these genes in sugar signal transduction and stress as well as sugar metabolism are discussed.

\section{Cross-Talk Between Hormone Biosynthesis and Sugar Signaling}

One of the five ESTs up-regulated in high sugar content (HS) mature leaves coded for an omega-3 fatty acid
desaturase-FAD8 (CA079174) (Table 1). In higher plants, the membrane lipids contain a high proportion of trienoic fatty acids (TAs). It has been suggested that these fatty acids, especially linolenic acid, are precursors of a defenserelated signal molecule, jasmonate (JA). In Arabidopsis, three genes encoding omega-3 fatty acid desaturase, namely $F A D 3, F A D 7$ and FAD8, are responsible for the production of TAs. The sugarcane gene FAD 8 was enriched in the high sugar content individuals 9 months after planting (Fig. 3), and was always more expressed in these plants throughout the growing season (Fig. 4). This EST was more expressed in immature leaves and also had high levels in immature internodes, which are considered as sink tissue, but also presented a weak expression in mature leaves (Fig. 5). Several enzymes envolved in methyl jasmonate biosynthesis were found to be more expressed in immature internodes, demonstrating that JA biosynthesis also seems to have a relevant role in culm development [38]. 
Fig. 3 Expression levels of differentially expressed genes in sugarcane individuals. RNA blots were prepared using $10 \mu \mathrm{g}$ of total RNA isolated from mature leaves of three individual clones of each segregated plants (HS - high and LS - low sugar contents). The time point evaluated in the blots corresponds to the same one used in the cDNA microarray experiments

(9 months after planting). Blots were hybridized with the genespecific radioactive probes indicated. An rDNA fragment was used as the control
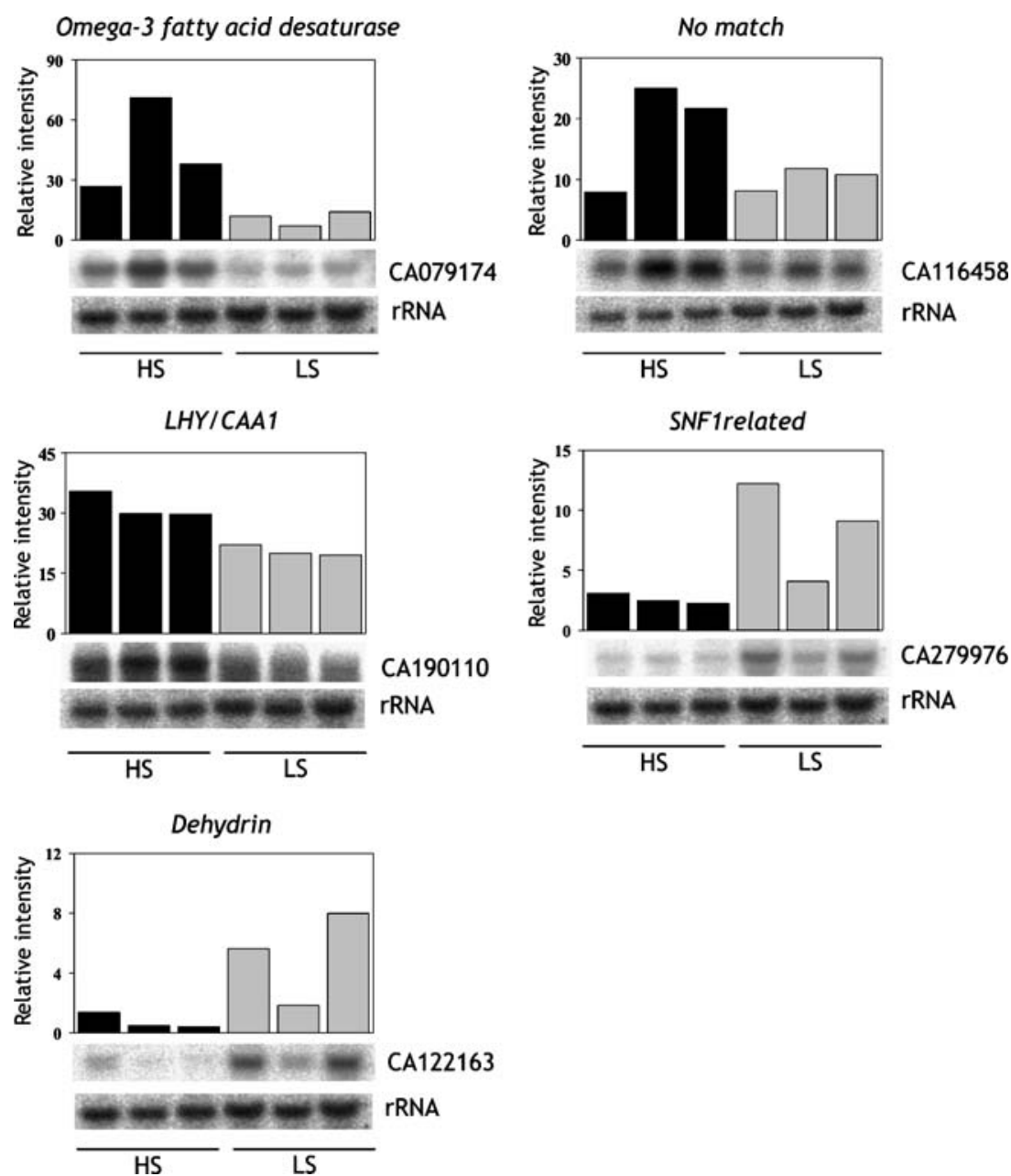

We also found evidence that the sugar-sensing and signal transduction systems interact with pathways responsive to other stimuli. The differentially expressed gene ScBAK1 (CA156919), a leucine-rich repeat receptor-like kinase, had sequence similarity to the brassinosteroid insensitive1associated receptor kinase. This gene was more expressed in high sugar content plants (this work) and in situ hybridization showed that its transcripts were preferentially expressed in the bundle-sheath cells and it was expressed only in mature leaves [54]. Due to the considerable sequence similarity between ScBAK1 and orthologues in sorghum and rice, it was suggested that the sugarcane protein was a component of a brassinosteroid receptor complex, and might play a role in brassinosteroid signaling.

Putative Model for Sugar Starvation Regulation of SPS

SnRK1 (SNF1-Related Protein Kinase-1) is a plant protein kinase with a catalytic domain similar to that of SNF1 (Sucrose Non-fermenting-1) of yeast and AMPK (AMPactivated protein kinase) of animals [16]. In plants, SNF1- related kinases have been named SnRK1 [14] and comprise three distinct sub-families (SnRK1, SnRK2 and SnRK3). Members of all three sub-families were identified in sugarcane [48]. Studies led to the hypothesis that once SnRK1 is activated in response to high intracellular sucrose and/or low intracellular glucose levels, SnRK1 can phosphorylate plant enzymes and activate starch synthesis in potato tubers $[16,44]$. The first plant protein to be identified as a substrate for SnRK1 was a HMG-CoA reductase in $A$. thaliana [10]. Subsequently, two other important enzymes, SPS and NR were shown to be substrates for SnRK1 phosphorylation in Ser-binding sites. In both cases, phosphorylation results in inactivation of the enzyme, although the inactivation of NR and SPS also requires the binding of a 14-3-3 protein to the phosphorylation site [2, 32].

A sugarcane $S n R K 1$ transcript (CA279976) (Table 1) was up-regulated in low sugar content mature leaves, 9 months after planting (Fig. 3). However it can be observed in Fig. 4 that, at times, this transcript had the opposite expression profile. For example, its levels were lower in the low sugar content leaves, 6, 11 and 13 months after planting. Transcripts 

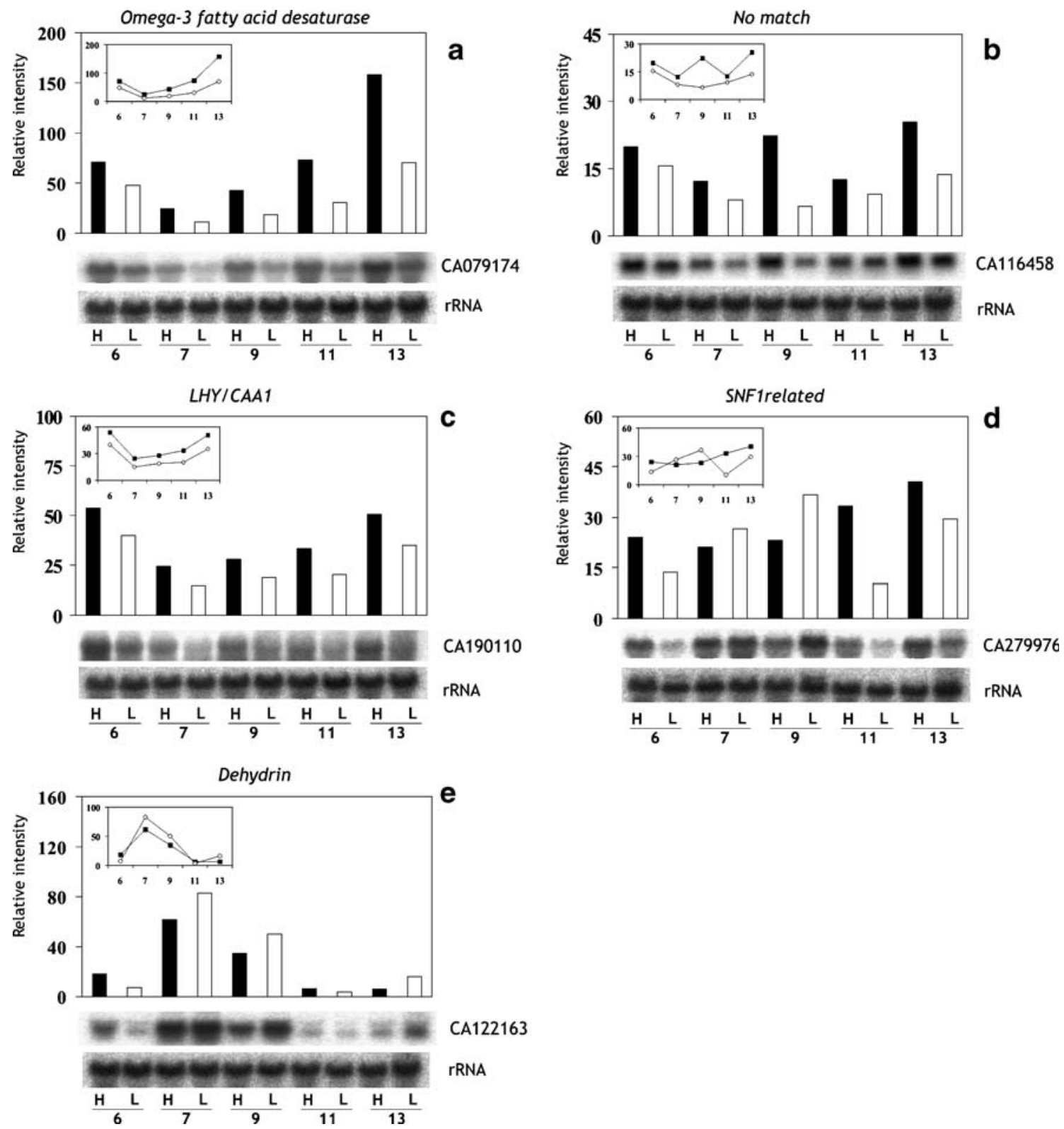

Fig. 4 Expression profiles of differentially expressed genes throughout the growing season. RNA-blots were prepared from total leafRNA from a pool of seven individuals with high (HS) and low (LS) sugar contents collected throughout the growing season $(6,7,9,11$

and 13 months after planting). The inset graphs show the expression levels observed for the high (black circles) and low (white circles) sugar content plants. An rDNA fragment was used as the control

of this gene had higher expression in sink tissues, such as immature leaves, internodes, lateral buds and flowers (Fig. 5), suggesting the involvement of this kinase in sugar translocation. Recently, McCormick et al. [28] artificially increase foliar sucrose content in field-grown sugarcane leaves using cold-girdling. Our data with the $S n R K 1$ gene is in agreement with their findings, since they observed a down-regulation of two genes encoding SnRK1-related proteins.

Three ESTs coding for 14-3-3 proteins (CA146811, $C A 132593$ and $C A 133114$ ) were found to be more expressed in mature leaves from the LS population (Table 1). Under the

conditions of low sugar content, SPS activity decreases because of an increase in the phosphorylation state of the enzyme ([18]; reviewed by Paul and Foyer [39]). Our previous work identified two ESTs coding for SnRKs proteins and four coding for 14-3-3 that were expressed at lower levels in mature internodes [38].

As stated above, the fact that three sugarcane 14-3-3 and a SnRK1 were more expressed in low sugar content individuals could reflect their role in keeping SPS in an inactivated state that would account for the lower sucrose levels in these plants. However, the expression profile along 


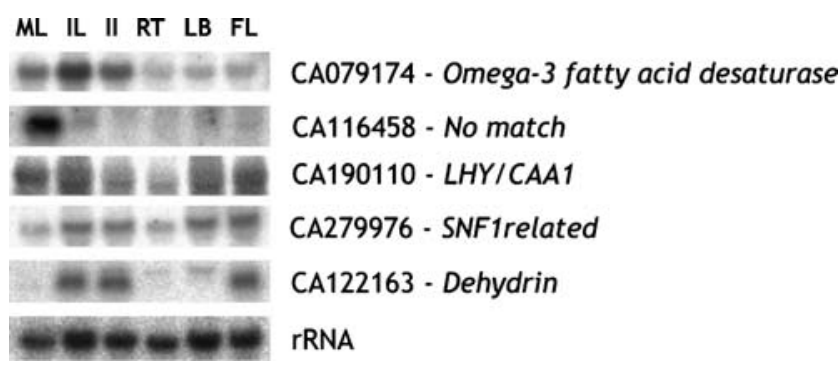

Fig. 5 Gene expression analysis in different tissues. For the RNA gel blot preparation, each lane was loaded with $10 \mu \mathrm{g}$ of total RNA isolated from one of six tissues from sugarcane. ML - mature leaves; IL-immature leaves; II-immature internode; RT—root; LB-lateral bud; FL-flowers. The same blot was hybridized to the indicated cDNA probes. An rDNA fragment was used as the control

the growing season observed for SnRK1 suggests that a complex regulation might be involved in the signaling pathway modulated by these genes. Future work with transgenic sugarcane plants would be helpful to discover the function of these genes. In fact, our preliminary results with transgenic sugarcane plants silenced for this gene had higher levels of sugars in their leaves (data not shown), which also reinforces the usefulness of the approach of using Bulk Segregant Analysis coupled to cDNA microarrays.

Lignin Biosynthesis and Secondary Wall Synthesis in Low Sugar Content Sugarcane Plants

Lignin is a complex polymer, which provides structural integrity in plants. In sugarcane bagasse it makes $23.1 \%$ by weight of biomass. Lignin remains as residual material after the sugars in the biomass have been converted to ethanol. It contains a lot of energy and can be burned to produce steam and electricity for the biomass-to-ethanol process. Three enzymes are involved in the biosynthetic pathway of lignin: cinnamoyl-coenzyme A reductase (CCR), cinnamyl alcohol dehydrogenase (CAD) and caffeic acid 3-O-methyltransferase (COMT). An EST coding for a COMT (CA125200) was found to be enriched in the low sugar content population (Table 1). The timing and localization of some of these genes show a strong correlation with the deposition of lignin, like in mature sugarcane stems [6]. The storage parenchyma of the maturing sugarcane stem internodes is extensively lignified and Jacobsen et al. [20] proposed that this process parallels with the increase in sucrose content observed in mature internodes. We observed that the LS plants had more lignified leaves and stem barks than HS plants (our unpublished data), in agreement with the higher levels of COMT transcripts in these plants. Interestingly, in transgenic alfalfa plants with reduced levels of COMT the cell walls were more amenable to enzymic degradation [8]. It is important to note that there is an association of COMT enzymes with sugar levels in sugarcane leaves (reported in this work) and internodes [38], making this gene a potentially valuable target for future genetic manipulation to increase sugarcane biomass.

\section{Expression of Stress-Related Proteins in Low Sugar Content Plants}

Sugar-signaling pathways do not operate in isolation but are part of cellular regulatory networks. Recent results clearly show cross-talk between different signaling systems, especially those of sugars, phytohormones and light. It is interesting to note that eight stress-related genes were upregulated in the LS plants. Most of them are cold and drought-induced and they were also previously identified in association with sucrose content in sugarcane internodes $[37,38,48]$.

Two sugarcane stress-related ESTs (CA186860 and CA160294) belong to a class of low-molecular-weight hydrophobic proteins involved in maintaining the integrity of the plasma membrane during cold, dehydration and salt stress conditions. These genes are activated by environmental factors, such as dehydration and salinity, and by chemical signals such as abscisic acid [33]. Another differentially expressed stress-related gene encodes a plasma membrane intrinsic protein (CA119392). These proteins facilitate water flux across cell membranes and play important roles in plant growth and development. Two ESTs coding for a dehydrin (CA124270 and CA122163) was also up-regulated in the LS plants (Figs. 3 and 4). These proteins are supposed to stabilize macromolecules and/or protect membranes against chilling damage [40]. Two putative sugarcane dehydrin-like proteins were identified by Nogueira et al. [34] in a sugarcane cold-response data mining. They proposed that these putative sugarcane antifreeze proteins could confer cellular membrane protection, reducing chilling injury.

The S-like RNase (CA135201) is a protein present in higher plants that controls self-incompatibility. In a selfincompatible Antirrhinum, S-RNAse transcription was induced during leaf senescence and phosphate (Pi) starvation but not by wounding, indicating that this gene plays a role in remobilizing $\mathrm{Pi}$ and other nutrients [24]. Finally, a stress-related EST differentially expressed in low sugar content plants, is a protein described as being woundinduced (CA127342).

Another large-scale analysis of gene expression in internodes of the same population segregating for Brix content that was used in this work also identified genes associated with sucrose content $[37,38]$. Intriguingly, as we observed in leaves, several proteins related to stress responses, such as dehydrin and low-molecular-weight 
hydrophobic proteins, were found to be differentially expressed in internodes [37, 38]. Approximately half of the sucrose content associated genes were found to be developmentally regulated during culm maturation, and many were related to stress responses. A comparison of this differential expression dataset with the results obtained when the plants were submitted to drought [48] revealed that approximately half of the genes identified as associated with the sucrose content were responsive to drought. They belonged to several functional categories including calcium signaling, stress responses, and protein phosphorylation.

These data indicated that the sucrose levels activate pathways during their synthesis in mature leaves and accumulation in internodes, which overlap with drought and other stress responses such as cold and injury.

\section{Concluding Remarks}

It is clear that sink tissues exert an influence on the photosynthetic rates and carbohydrate levels of source organs [27, 39]. A relationship between source and sink tissues was demonstrated in sugarcane [27], where demand for carbon from sinks tissues affects source leaf photosynthetic activity, metabolite levels and also the gene expression.

In fact, the activity of photosynthesis-related enzymes and the expression of their genes in the source leaf are modified by the local levels of sugar and hexoses that will be transported to sink [43]. As observed in sugarcane, decreased hexose levels in leaves may act a signal for increased sink demand, reducing a negative feedback regulation of photosynthesis [26], and hexoses, rather than sucrose, could be involved in this regulation [27]. In the same way, our results indicates a negative correlation of hexose levels between source leaves and mature internode of HS and LS plants, (Fig. 2). Hexoses have been involved in the regulation of source metabolism via signal transduction pathways involving protein phosphorylation by MAPK activities [11]. However it is difficult to address the specific role of this enzyme in the source to sink regulation. There is a wide variety of signaling pathways associated with these kinases, and the same occur to many other components related to signal transduction.

Interestingly, the gene expression profiles along the growing season did not point to any pathway that was activated only once during the maturation process This is in line with the complexity of the sucrose accumulation process in sugarcane, and indicates that the activation of some signal transduction components may be needed during long periods.
As stated above, the expression of 14-3-3 and a SnRK1 give insights in a feedback regulation of photosynthesis, keeping the enzyme SPS in an inactivated state that would account for the lower sucrose levels in these plants. These results are consistent those observed in sugarcane [27, 28] and the notion that sink demand may limit source activity through a kinase-mediated sugar signaling mechanism that correlates to a decrease in source hexose concentrations.

Our results also suggests that sugar levels seem to modulate gene expression at the transcriptional level through a complex signal transduction network that may involve common responses related to stress. The data provide an insight into the role of sugar levels in signal transduction pathways. Some expression trends of low sugar levels such as up-regulation of 14-3-3 proteins, a SnRK1 and stress-related proteins were substantiated by the present data at the transcript level. These genes are interesting targets for further research using other approaches, such as overexpression or gene silencing. An in-depth analysis of these components should improve our knowledge on how signal transduction can regulate sucrose synthesis in sugarcane plants.

\section{Methods}

\section{SUCAST Catalogue Annotation}

A comprehensive sugarcane EST (Expressed Sequence Tags) data collection was made available by the SUCEST Consortium in 2003 [53]—http://sucest.lad.ic.unicamp.br/ public) and functional characterization of molecular components is underway (http://www.sucest-fun.org). A total of 43,141 Sugarcane Assembled Sequences (SAS) representing the putative transcripts from sugarcane have been found. A subset of 902 transcripts related to elements of signaling cascades, transcription factors and stress-related transcripts, in particular, plus 378 transcripts encoding proteins with unknown function are the focus of this work and have been described previously [36-38, 47, 48].

\section{cDNA Microarrays}

Microarrays were constructed by arraying 1920 PCRamplified cDNA fragments on derivative glass slides as described by Papini-Terzi et al. [36]. Four replicates of each cDNA fragment were distributed across each array. Fragments for which the amplification reactions were not satisfactory or hybridization signals were low were removed from the analysis. High quality data was obtained for a total of 1280 SAS, all of which had their identity confirmed by re-sequencing. 


\section{Sugarcane Tissue Samples}

Sugarcane F1 plants were obtained from a cross between pre-commercial Brazilian cultivars (SP80-180 X SP804966). The population is comprised of 498 individuals that segregated for stem sugar content in a normal manner and was previously described by Garcia et al. [12] and PapiniTerzi et al. [37]. The seven plants presenting extreme values for high sugar (HS) and low sugar (LS) were selected. Mature leaves (Leaf +1 , according to [51]) were collected from the selected plants $6,7,9,11$ and 13 months after planting-February, March, May, July and September, respectively. To avoid the effect of diurnal rhythms all samples were collected around the same time: $9 \mathrm{~h} 00 \mathrm{am} \pm 30$ $\min$. For the microarray analyses, leaves collected at the 9 months time point from each of the seven individuals of each group were pooled and used for RNA extraction. The expression profiles observed in the microarrays were further validated by RNA blot using RNA from three HS and three LS individuals collected at the 9 months time point. Pooled RNA from the seven HS and LS individuals collected at all five time points were also used in RNA blots to detect the expression profiles along the growing season. The expression profile of selected genes was also evaluated for six different tissues collected from 12 month old plants: mature leaf, immature leaf, immature internode, root, lateral bud and a mixture of flowers in different developmental stages, using the same commercial sugarcane varieties used in the SUCEST project (SP87-432 for flowers and SP803280 for other tissues). All tissue samples were stored at $-80^{\circ} \mathrm{C}$.

Sugar Measurement in Leaves and Stem

Two measurements were taken for sugars content: one for Brix analysis in mature internodes and the other for HPLC and HPAEC-PAD analysis in mature leaves and internodes according to Clarke et al. (1983) [9]) and Tai and Miller [49]. The soluble solids (Brix) content of each sugarcane segregant plant (HS and LS) was measured on a portable refractometer (N1 model, ATAGO, Japan) throughout the growing season. For parental plants the Brix content was measured only in September (13 months after planting). This analysis was carried out immediately after the cane sample was crushed. Mature leaves and the most mature internode from three individual clones of each segregated plants (HS and LS) were chosen for xylitol, mannitol, trehalose, arabinose, galactose, glucose, fructose, maltose and sucrose contents. Sucrose was measured using HPLC and the other sugars were measured by HPAEC-PAD (High Performance Anion Exchange Chromatography). These sugar measurements were done at 9 months after planting, that was the same time point used for microarray analysis.

\section{RNA Extraction}

Leaf tissue (2-2.5 g) was ground to a fine powder in liquid nitrogen, using pre-cooled mortar and pestle. RNA was isolated using the $\operatorname{Trizol}^{\circledR}$ reagent (Invitrogen, USA), following the recommended procedure. The RNA samples were quantified in a spectrophotometer and loaded onto $1.0 \%$ agarose/formaldehyde gels for a quality inspection. RNA samples of five sugarcane tissues (flower, leaf, stem, root and bud) were also prepared and equimolarly mixed, to be used in homotypic (self-self) hybridizations. The Trizol ${ }^{\circledR}$ manufacturer's recommendations for high polysaccharide content tissues were followed for the mature internode samples.

\section{Probe Preparation and Hybridization}

Two microarray hybridizations (Lv1 and Lv2) were performed comparing one pooled sample from seven plants with high sugar content (HS) to another pool from seven plants with low sugar content (LS), in a dye-swap layout. RNA samples for Lv1 and Lv2 hybridizations derived from independent extractions from the same pools of plants.

To this end, ten micrograms of total RNA were reverse transcribed using oligo dT primers and labeled using the CyScribe Post-Labeling kit (Amersham Biosciences, Sweden), according to the manufacturer's instructions. The products of the labeling reactions were purified in filtering plates (Multiscreen MAFBN0B50, Millipore, USA) to remove unincorporated labeled nucleotides. The microarrays were co-hybridized with the fluorescently labeled probes. Hybridizations were performed overnight at $42^{\circ} \mathrm{C}$ in moist chambers. The slides were then washed in $1 \mathrm{x}$ SSC and $0.2 \% \operatorname{SDS}\left(10 \mathrm{~min}, 55^{\circ} \mathrm{C}\right)$, twice in $0.1 \mathrm{x} \mathrm{SSC}$ and $0.2 \%$ SDS $\left(10 \mathrm{~min}, 55^{\circ} \mathrm{C}\right)$ and finally in $0.1 \times \mathrm{SSC}(1 \mathrm{~min}, \mathrm{RT})$. The slides were rinsed briefly in filtered milli-Q water and dried in a nitrogen stream. Each experimental step was carefully monitored to ensure high quality of the slides and the extracted data.

\section{Data Extraction and Processing}

The slides were scanned using a Generation III Scanner ${ }^{\mathrm{TM}}$ (Molecular Dynamics, USA) and processed using the ArrayVision (Imaging Research Inc., Canada) software. Low-quality spots were filtered. Signal intensities were calculated for each valid spot subtracting the local median background from the MTM (median-based trimmed mean) density. 
The raw fluorescence intensity values were then processed using custom programs on $\mathrm{R}$ language [19], available at http://verjo19.iq.usp.br/xylella/microarray). Firstly, intensity ratios (HS/LS) were calculated for each spot. Then, each slide dataset was normalized using the Lowess fitting [55], in order to correct for systematic experimental errors such as labeling-bias and intensity dependent variation. To be able to classify a gene as differentially expressed, a set of experimental and computational steps was established, using a local implementation of the HTself method [52], as follows: 1) Homotypic or "selfself" hybridizations were performed using a tissue-pool sample in both channels (Cy3 and $\mathrm{Cy} 5)$ to assess experimental "noise", i.e., the intrinsic technical variation of the experimental pipeline; 2) The fluctuation of the normalized ratios obtained from these homotypic hybridizations was computed in an intensity-dependent manner, integrating the probability density function to $98 \%$ for eight different signal intensity intervals. Thus, a ratio cut-off curve that determines the limits of the random variation for our data could be outlined; 3) The replicate ratio values obtained for each gene were independently compared to the cut-off limits and classified as up (above the cut-off limit), down (below the cut-off) or inside (no differential expression). Genes with at least $75 \%$ of the replicate points above or below (up or down) the cut-off limits were considered differentially expressed.

The ratios obtained for each transcript in our chip can be found in the supplemental material (Table S-1). Descriptions followed the MIAME guidelines and the data was deposited on Gene Expression Omnibus database (GEO-http:// www.ncbi.nlm.nih.gov/geo/) under the accession numbers GSE4233 (series), GPL1376 (platform), GSM95526, GSM95546, GSM95547 and GSM95548 (samples).

\section{Validation of Microarray Results by RNA Blot}

Electrophoresis of total RNA samples $(10 \mu \mathrm{g})$ was carried out on $1.5 \%$ formaldehyde-containing agarose gels by standard procedures [45] and transferred to a nylon filter (Hybond- $\mathrm{N}^{+}$, Amersham Biosciences, Sweden). For each gene tested, the longest EST clone of each SUCEST SAS was selected as a probe for RNA blot hybridization. Inserts were labeled with the Read-To-Go kit (Amersham Biosciences, USA) according to the protocol recommended by the manufacturer. Hybridized filters were exposed to imaging plates for $24 \mathrm{~h}$ and the digitized images of RNA blot hybridization signals detected using the FLA3000-G screen system (Fuji Photo Film, Japan) and quantified using the Image Gauge software v. 3.12 (Fuji Photo Film, Japan).

Acknowledgments We are indebted to Adriana Y. Matsukuma and Denise Yamamoto for their technical assistance in microarray printing and scanning performed in the laboratory of the Cooperation for Analysis of Gene Expression (CAGE) and Dr. Sonia di Mauro for coordinating the distribution of the SUCEST clones. We are also indebted to Sabrina Moutinho Chabregas, Paulo Gouvêa and William Burnquist for their technical support in the development of this work. This work was funded by FAPESP (Fundação de Amparo à Pesquisa do Estado de São Paulo) and CNPq.

\section{References}

1. Altschul SF, Madden TL, Schaffer AA, Zhang JH, Zhang Z, Miller W, Lipman DJ (1997) Gapped BLAST and PSI-BLAST: a new generation of protein database search programs. Nucleic Acid Res 25:3389-3402

2. Bachmann M, Huber JL, Athwal GS, Wu K, Ferl RJ, Huber SC (1996) 14-3-3 proteins associate with the regulatory phosphorylation site of spinach leaf nitrate reductase in an isoform-specific manner and reduce dephosphorylation of Ser-543 by endogenous protein phosphatases. FEBS Lett 398:26-30

3. Carson DL, Botha FC (2002) Genes expressed in sugarcane maturing internodal tissue. Plant Cell Rep 20:1075-1081

4. Carson DL, Huckett BI, Botha FC (2002) Sugarcane ESTs differentially expressed in immature and maturing internodal tissue. Plant Sci 162:289-300

5. Casu RE, Grof CPL, Rae AL, McIntyre CL, Dimmock CM, Manners JM (2003) Identification of a novel sugar transporter homologue strongly expressed in maturing stem vascular tissues of sugarcane by expressed sequence tag and microarray analysis. Plant Mol Biol 52:371-386

6. Casu RE, Dimmock C, Chapman S, Grof C, McIntyre CL, Bonnett G, Manners J (2004) Identification of differentially expressed transcripts from maturing stem of sugarcane by in silico analysis of stem expressed sequence tags and gene expression profiling. Plant Mol Biol 54:503-517

7. Casu RE, Manners JM, Bonnett GD, Jackson PA, McIntyre CL, Dunne R, Chapman SC, Rae AL, Grof CPL (2005) Genomics approaches for the identification of genes determining important traits in sugarcane. Field Crop Res 92:137-147

8. Chen F, Dixon R (2007) Lignin modification improves fermentable sugar yields for biofuel production. Nat Biotechnol 25:759-761

9. Clarke MA, Tsang WSC, Parrish FW (1983) High performance liquid chromatography in sugar factories and refineries. In: Proc Sugar Ind Technol 42nd Annual Meeting, Oak Harbor, WA, p $121-142$

10. Dale S, Arro M, Becerra B, Morrice NG, Boronat A, Hardie DG, Ferrer A (1995) Bacterial expression of the catalytic domain of 3hydroxy-3- methylglutaryl-CoA reductase (isoform HMGR1) from Arabidopsis thaliana, and its inactivation by phosphorylation at Ser577 by Brassica oleracea 3-hydroxy-3-methylglutaryl-CoA reductase kinase. Eur J Biochem 233:506-513

11. Ehness R, Ecker M, Godt D, Roitsch T (1997) Glucose and stress independently regulate source/sink relations and defense mechanisms via signal transduction pathways involving protein phopsphorylation. The Plant Cell 9:1825-1841

12. Garcia AAF, Kido EA, Meza AN, Souza HMB, Pinto LR, Pastina MM, Leite CS, da Silva JAG, Ulian EC, Figueira A, Souza AP (2006) Development of an integrated genetic map of a sugarcane (Saccharum spp ) commercial cross, based on a maximumlikelihood approach for estimation of linkage and linkage phases. Theor Appl Genet 112:298-314

13. Gibson SI (2005) Control of plant development and gene expression by sugar signaling. Curr Opin Plant Biol 8:93-102 
14. Halford NG, Hardie DG (1998) SNF1-related protein kinases: global regulators of carbon metabolism in plants? Plant Mol Biol 37:735-748

15. Halford NG, Paul MJ (2003) Carbon metabolite sensing and signaling. Plant Biotechnol J 1:381-398

16. Halford NG, Hey S, Jhurreea D, Laurie S, McKibbin RS, Paul M, Zhang Y (2003) Metabolic signaling and carbon partitioning: role of Snf1-related (SnRK1) protein kinase. J Exp Bot 54:467-475

17. Ho SL, Chao YC, Tong WF, Yu SM (2001) Sugar coordinately and differentially regulates growth- and stress-related gene expression via a complex signal transduction network and multiple control mechanisms. Plant Physiol 125:877-890

18. Huber JL, Huber SC, Nielsen TH (1989) Protein phosphorylation as a mechanism for regulation of spinach leaf sucrose-phosphate synthase activity. Arch Biochem Biophys 270:681-690

19. Ihaka R, Gentleman R (1996) R: A language for data analysis and graphics. J Comput Graph Stat 5:299-314

20. Jacobsen KR, Fisher DG, Maretzki A, Moore PH (1992) Developmental-changes in the anatomy of the sugarcane stem in relation to phloem unloading and sucrose storage. Botanica Acta 105:70-80

21. Jansen RC, Nap JP (2001) Genetical genomics: the added value from segregation. Trends Genet 17:388-391

22. Koch KE (1996) Carbohydrate-modulated gene expression in plants. Annu Rev Plant Physiol Plant Mol Biol 47:509-540

23. Koch KE (2004) Sucrose metabolism: regulatory mechanisms and pivotal roles in sugar sensing and plant development. Curr Opin Plant Biol 7:235-246

24. Liang LZ, Lai Z, Ma WS, Zhang YS, Xue YB (2002) AhSL28, a senescence- and phosphate starvation-induced S-like RNase gene in Antirrhinum. BBA - Gene Struct Expr 1579:64-71

25. Lunn JE, Furbank RT (1999) Sucrose biosynthesis in C-4 plants. New Phytol 143:221-237

26. McCormick AJ, Cramer MD, Watt DA (2006) Sink strength regulates photosynthesis in sugarcane. New Phytol 171:759-770

27. McCormick AJ, Cramer MD, Watt DA (2007) Changes in photosynthetic rates and gene expression of leaves during a source-sink perturbation in sugarcane. Ann Bot 101:89-102

28. McCormick AJ, Cramer MD, Watt DA (2008) Differential expression of genes in the leaves of sugarcane in response to sugar accumulation. Trop Plant Biol 1:142-158. doi:10.1007/ s12042-008-9013-2

29. Menossi M, Silva-Filho MC, Vincentz M, Van-Sluys MA, Souza GM (2008) Sugarcane functional genomics: gene discovery for agronomic trait development. Int J Plant Genomics . doi:10.1155/ 2008/458732

30. Meyers BC, Galbraith DW, Nelson T, Agrawal V (2004) Methods for transcriptional profiling in plants. Be fruitful and replicate. Plant Physiol 135:637-652

31. Moore PH (2005) Integration of sucrose accumulation processes across hierarchical scales: towards developing an understanding of the gene-to-crop continuum. Field Crops Res 92:119-135

32. Moorhead G, Douglas P, Cotelle V, Harthill J, Morrice N, Meek S, Deiting U, Stitt M, Scarabel M, Aitken A, MacKintosh C (1999) Phosphorylation-dependent interactions between enzymes of plant metabolism and 14-3-3 proteins. Plant J 18:1-12

33. Morsy MR, Almutairi AM, Gibbons J, Yun SJ, los Reyes BG (2005) The OsLti6 genes encoding low-molecular-weight membrane proteins are differentially expressed in rice cultivars with contrasting sensitivity to low temperature. Gene 344:171-180

34. Nogueira FTS, De Rosa VE, Menossi M, Ulian EC, Arruda P (2003) RNA expression profiles and data mining of sugarcane response to low temperature. Plant Physiol 132:1811-1824

35. Osuna D, Usadel B, Morcuende R, Gibon Y, Bläsing OE, Höhne M, Günter M, Kamlage B, Trethewey R, Scheible W-R, Stitt M (2007) Temporal responses of transcripts, enzyme activities and metabolites after adding sucrose to carbon-deprived Arabidopsis seedlings. Plant J 49:463-491

36. Papini-Terzi FS, Rocha FR, Vencio ZN, Oliveira KC, Felix JM, Vicentini R, Rocha CD, Simoes ACQ, Ulian EC, Di Mauro SMZ, Da Silva AM, Pereira CAD, Menossi M, Souza GM (2005) Transcription profiling of signal transduction-related genes in sugarcane tissues. DNA Res 12:27-38

37. Papini-Terzi FS, Felix JM, Rocha FR, Waclawovsky AJ, Ulian EC, Chabregas SM, Falco MC, Nishiyama-Junior MY, Vencio RZN, Vicentini R, Menossi M. and Souza GM (2007) The SUCEST-FUN Project: identifying genes that regulate sucrose content in sugarcane plants. In: XXVI International Society of Sugarcane Technologists Congress, 2007, Durban. Proc. Int. Soc. Sugar Cane Technol., vol 26

38. Papini-Terzi FS, Rocha FR, Vêncio RZN, Felix JM, Branco DS, Waclawovsky AJ, Del Bem LEV, Lembke CG, Costa MDL, Nishiyama MY Jr, Vicentini R, Vincentz MGA, Ulian EC, Menossi M, Souza GN (2009) Sugarcane genes associated with sucrose content. BMC Genomics 10:120

39. Paul MJ, Foyer CH (2001) Sink regulation of photosynthesis. J Exp Bot 52:1383-1400

40. Pearce RS (1999) Molecular analysis of acclimation to cold. Plant Growth Regul 29:47-76

41. Pessoa Junior A, Roberto IC, Menossi M, Santos RR, Ortega Filho S, Penna TCV (2005) Perspectives on bioenergy and biotechnology in Brazil. Appl Biochem Biotechnol 121:59-70

42. Price J, Laxmi A, St Martin SK, Jang JC (2004) Global transcription profiling reveals multiple sugar signal transduction mechanisms in Arabidopsis. Plant Cell 16:2128-2150

43. Rolland F, Moore B, Sheen J (2002) Sugar sensing and signaling in plants. Plant Cell 14:S185-S205

44. Rolland F, Baena-Gonzalez E, Sheen J (2006) Sugar sensing and signaling in plants: conserved and novel mechanisms. Annu Rev Plant Biol 57:675-709

45. Sambrook J, Fritsch EF, Maniats T (1989) Molecular cloning: A laboratory manual. Cold Spring Harbor, New York

46. Smeekens S (2000) Sugar-induced signal transduction in plants. Annu Rev Plant Physiol Plant Mol Biol 51:49-81

47. Souza GM, Simoes ACQ, Oliveira KC, Garay HM, Fiorini LC, Gomes FD, Nishiyama-Junior MY, da Silva AM (2001) The sugarcane signal transduction (SUCAST) catalogue: prospecting signal transduction in sugarcane. Genet Mol Biol 24:25-34

48. Rocha FR et al (2007) Signal transduction-related responses to phytohormones and environmental challenges in sugarcane. BMC Genomics 8:71

49. Tai PYP, Miller JD (2002) Germplasm diversity among four sugarcane species for sugar composition. Crop Sci 42:958-964

50. Thum K, Shin M, Palenchar P, Kouranov A, Coruzzi G (2004) Genome-wide investigation of light and carbon signaling interactions in Arabidopsis. Genome Biol 5:R10

51. Van Dillewijn C (1952) Botany of sugarcane. Walthan Mass, ed (USA)

52. Vencio RZN, Koide T (2005) HTself: Self-Self based statistical test for low replication microarray studies. DNA Res 12:211-214

53. Vettore AL, da Silva FR, Kemper EL, Souza GM, da Silva AM et al (2003) Analysis and functional annotation of an expressed sequence tag collection for tropical crop sugarcane. Genome Res 13:2725-2735

54. Vicentini R, de Maria Felix J, Dornelas MC, Menossi M (2009) Characterization of a sugarcane (Saccharum spp.) gene homolog to the brassinosteroid insensitive1-associated receptor kinase 1 that is associated to sugar content. Plant Cell Rep 28:481-491

55. Yang YH, Dudoit S, Luu P, Lin DM, Peng V, Ngai J, Speed TP (2002) Normalization for cDNA microarray data: a robust composite method addressing single and multiple slide systematic variation. Nucleic Acids Res 30:e15

56. Yu SM (1999) Cellular and genetic responses of plants to sugar starvation. Plant Physiol 121:687-693 